\title{
Resiliencia comunitaria frente a la exclusión social: caso María Cano Carambolas, Medellín
} Community resilience facing social
exclusion: a case study in Maria
Cano Carambolas, Medellin

Clara Acuña Rodríguez, Inés Lasagabaster Unzueta**

Recibido: 25 de noviembre de 2020 / Aceptado: 8 de julio de 2021 / Publicado: 13 de diciembre de 2021

Forma de citar este artículo en APA:

Acuña Rodríguez, C., \& Lasagabaster Unzueta, I. (2021). Resiliencia comunitaria frente a la exclusión social: caso María Cano Carambolas, Medellín. Poiésis, (41), 43-63. https://doi.org/10.21501/16920945.4187

\section{Resumen}

Este artículo tuvo como propósito crear un espacio de reflexión acerca de las capacidades resilientes de los habitantes del barrio María Cano Carambolas (Medellín-Colombia) a partir de observaciones participantes. El trabajo de campo se documentó mediante diarios de campo y relatos del alumnado de la principal institución educativa. A partir del estudio de las condiciones socio-económicas, políticas y educativas del barrio, analizaremos las habilidades de afrontamiento y las estrategias resilientes comunitarias. Nuestras conclusiones resaltan la alta capacidad de abstracción ante las problemáticas que les rodean y en el caso de los jóvenes, la percepción positiva de logro de objetivos a medio-largo plazo; a pesar de que las dinámicas disfuncionales de sus familias, las políticas públicas insuficientes e ineficaces de un Estado negligente y las propias condiciones del barrio limitan su acceso a oportunidades de futuro.

\section{Palabras clave:}

Colombia; Comunidad; Educación; Estado; Exclusión social; Políticas públicas; Resiliencia.

Este artículo de reflexión surge del trabajo de campo realizado en el marco del proyecto de intervención social Cultura Amigó y esté vinculado al grupo de investigación "Familia, desarrollo y calidad de vida" de la Universidad Católica Luis Amigó (Colombia) como parte de una pasantía internacional entre dicha institución y la Universidad de Deusto (España) entre los meses de agosto y noviembre del 2018. Magíster en Psicología de la Intervención Social y Mediación Familiar, Universidad de Deusto, Bilbao-España. Contacto: clara.acuna@ opendeusto.es, [D https://orcid.org/0000-0002-8875-4362

Magíster en Psicología Jurídica; Psicología de la Intervención Social y Mediación Familiar, Universidad de Deusto, Bilbao-España Contacto: i.lasagabaster@opendeusto.es, [i] https://orcid.org/0000-0001-7634-4318 


\section{Abstract}

The purpose of this article was to create a reflection space on the resilient capacities of the inhabitants of the María Cano Carambolas neighborhood (Medellín-Colombia) based on participant observations. The field work was documented through field diaries and stories from the students of the main educational institution. From the study of the socio-economic, political and educational conditions of the neighborhood, we will analyze coping skills and community resilient strategies. Our conclusions highlight the high capacity for abstraction in the face of the problems that surround them and in the case of young people, the positive perception of achievement of medium-longterm objectives; despite the fact that the dysfunctional dynamics of their families, the insufficient and ineffective public policies of a negligent state and the very conditions of the neighborhood limit their access to future opportunities.

\section{Keywords:}

Colombia; Community; Education; State; Social exclusion; Public policies; Resilience. 


\section{Introducción}

Desde la gestación y primera infancia hasta la muerte, nuestro desarrollo integral como individuos se produce mediante el abordaje de tareas vitales y desafíos críticos, moldeado y construido en la relación y comunicación con otros agentes, instituciones y referentes significativos culturalmente (Phillips \& Shonkoff, 2000).

En el barrio María Cano Carambolas, situado en la Comuna 3 de la ciudad de Medellín, el contexto de desarrollo de los menores de la comunidad y sus familias es el de la exclusión social en todos sus vértices: estigmatización, indiferencia y rechazo social, originadas por la negligencia de las políticas del Estado Colombiano que no protegen los derechos y oportunidades de la ciudadanía por igual. Esta praxis podría resultar únicamente en el estancamiento de la pobreza y la indefensión aprendida de estas comunidades, las cuales difícilmente tienen la oportunidad de vislumbrar un porvenir prometedor. Sin embargo, comprobamos que a nivel generalizado sucede un fenómeno completamente opuesto, objeto de estudio de esta investigación. Menores y adultos se proyectan a un futuro de oportunidades y a la consecución de elevadas metas profesionales y familiares.

En definitiva, hacemos referencia a la resiliencia en familias multiproblemáticas en contextos vulnerables, definida por Gómez y Kotliarenco (2010) como "un engranaje relacional y eco-sistémico que permite encontrar oportunidades donde podría darse el estancamiento o deterioro" (p. 105).

\section{El barrio: contexto socioeconómico y funcionamiento comunitario}

María Cano Carambolas, territorio con más de 56 años de antigüedad, se ubica en la franja alta de la comuna 3, Manrique, en la periferia nororiental de Medellín. Se trata un barrio de estratos socioeconómicos 1, 2 y 3, en una escala de clasificación 1-6, que cuenta con una población diversa proveniente de distintas olas de desplazamiento forzoso rural, urbano e interurbano que han generado el asentamiento de colonias enteras en el departamento de Antioquia, cuya capital es Medellín, y otras partes del país.

El barrio ha vivido un crecimiento exponencial; en 2005 contaba con 2571 habitantes y solo ocho años después duplicaba su población con 6760. En 2013 la población infantil (0-14 años) era el grupo poblacional más numeroso, seguido de la población juvenil (14-28 años), que es considerada según estadísticas nacionales y urbanas como la más vulnerable en los ámbitos social y económico (Alcaldía de Medellín y Universidad de Antioquia, 2014). Este aumento poblacional y su carácter de asentamiento provisional han provocado la disminución de la calidad de vida en los últimos años. 
El último informe llevado a cabo entre 2016 y 2019 califica a Manrique como la tercera comuna de Medellín con el Índice Multidimensional de Calidad de Vida más bajo de la ciudad (Medellín Cómo Vamos, 2020). Este índice tiene en cuenta: el material de las viviendas, la presencia de toma de agua, el manejo de desechos sólidos, la presencia de servicios sanitarios y electrodomésticos en los hogares, el número de vehículos, el nivel de escolaridad de los progenitores, la proporción de menores sin escolarizar, personas analfabetas y con seguridad social y los niveles de hacinamiento.

Las problemáticas se concentran especialmente en la franja alta de la comuna, donde se ubica María Cano Carambolas. Los Planes de Desarrollo Local para la Comuna 3-Manrique (Alcaldía de Medellín, 2014) recogen las principales dificultades reportadas por la comunidad. Las que afectan a la población juvenil son: uso y abuso de sustancias psicoactivas, falta de oportunidades laborales, embarazo precoz y carencias en la oferta de actividades de ocio y tiempo libre. Según el Primer Estudio Poblacional de Salud Mental en Medellín, realizado entre 2011 y 2012, Manrique es catalogada como una comuna de riesgo muy alto para grupos de trastornos de ansiedad, afectivos, impulsivos y consumo de sustancias (Universidad CES y Secretaria de Salud de Medellín, 2012).

Por otra parte, la imposibilidad de acceder a un empleo formal e ingresos fijos acrecienta la situación de inestabilidad de las familias. La principal actividad económica de Manrique es el pequeño comercio y los negocios familiares. Dichos ingresos se invierten principalmente en alimentación y transporte y, su generación recae mayoritariamente sobre los hombres, ya que el empleo es más accesible y está mejor remunerado para ellos (Alcaldía de Medellín, 2014).

Se habla de riqueza de un país cuando las personas tienen oportunidades y acceden a ellas; y así el barrio de Carambolas, es pobre en todos los sentidos. Sus habitantes constan en el Sistema de Selección de Beneficiarios para Programas Sociales (Sisbén) y en el censo del Departamento Administrativo Nacional de Estadística. Están presuntamente incluidos en los programas de protección a desplazados, víctimas del conflicto armado y personas con discapacidad, lo que les daría prioridad en los servicios sociales y derecho a una cobertura sanitaria total.

Sin embargo, los habitantes refieren que sus altos puntajes en el Sisbén no corresponden a la situación real, impidiéndoles acceder a los beneficios que ofrece el Estado. Se encuentran en una situación de absoluta precariedad, ya que, aunque formalmente se reconozca su derecho a los servicios básicos, en la práctica no existe. Además, también describen problemas como la falta de atención e intervención que conducen a la desprotección social, la invisibilización de las víctimas del conflicto armado, la insuficiencia de los paquetes alimentarios de la Administración Municipal, la situación de abandono y marginalidad de la población más envejecida y en condición de discapacidad, así como el desconocimiento generalizado de los programas y puntos de salud ofertados (Alcaldía de Medellín, 2014).

El índice de participación electoral en el barrio, y en la comuna en general, no alcanzó el 60\%, siendo de los más bajos de la ciudad (Censo División Política de Medellín, 2018). Cabe cuestionar hasta qué punto, se debe a: dificultades estructurales de acceso al voto, una pobre conciencia sobre el ejercicio de este derecho 0 a la desesperanza ante la situación nacional. Estos factores 
se suman al bajo nivel de confianza en la clase política tras las situaciones de violencia e injusticia ejercidas sobre ellos. Así, se crea un círculo vicioso en el que la falta de participación, genera falta de oportunidades, que a su vez reducen las posibilidades de futuro y las esperanzas de que la participación política sea una herramienta de cambio.

Sin embargo, también hay formas alternativas de ejercer su poder de decisión a nivel micro. Es destacable la organización de los habitantes del barrio, generalmente de edad más avanzada, en sus propios grupos de liderazgo comunitarios independientes entre los que destaca la Junta de Acción Comunal (JAC). Este es un ejemplo de responsabilidad de la propia comunidad, que trabaja por el desarrollo de su barrio y territorio, poniendo de manifiesto una actitud de resistencia ante las adversidades en el acceso real a los mecanismos de decisión pública.

\section{Perfil y contexto del alumnado de la Institución Educautiva Reino de Bélgica (1.E.R.B)}

Para llegar a una comprensión integral de la comunidad, consideramos importante incluir una perspectiva generacional, por lo que nos acercamos a los jóvenes a través de la Institución Educativa Reino de Bélgica (I.E.R.B). Este es uno de los dos centros educativos públicos y gratuitos presentes en el barrio María Cano Carambolas. La escuela cuenta con 904 alumnos; el 94,7\% se declara de etnia mestiza y el 5,3\% restante afrodescendiente. En el I.E.R.B existe una sobrepoblación en las aulas (hasta 40 estudiantes en cada una), mezcla de edades diversas (hasta 4 años de diferencia) y un elevado absentismo escolar.

Otra problemática es la baja tasa de escolarización y el alto porcentaje de abandono. Los índices de deserción entre la población estudiantil, principalmente entre los 15-16 años, disminuyeron entre los años 2012 (3.67\%) y 2013 (2.5\%) (Alcaldía de Medellín, 2014). Sin embargo, aunque los datos indiquen una mejora de la cobertura y el acceso a la educación, no se incrementa su calidad, ya que no se invierten recursos para evitar el exceso de estudiantes por aula.

Las dificultades de accesibilidad afectan principalmente a la juventud de los barrios de la franja media-alta de la comuna, ya que las instituciones educativas cercanas no cuentan con las suficientes plazas y se ven obligados a desplazarse a otras que se ubican en barrios lejanos y de difícil acceso.

Por otro lado, el contexto familiar juega un papel esencial en el desarrollo de cada individuo y en este caso la gran mayoría de los menores del barrio provienen de familias multiproblemáticas. En la descripción de este patrón de contexto familiar se pueden encontrar las siguientes características (Gómez et al., 2007):

1. Problemáticas familiares, de naturaleza compleja y diversa (contextos violentos, marginación social etc.) que derivan en crisis recurrentes.

2. Ausencia de roles, rutinas y normas definidas, desorganización generalizada y falta de comunicación, debido a la ausencia de tiempo y a los elevados niveles de estrés. 
3. La negligencia y abandono de las funciones parentales dificulta la adaptación e integración de los hijos y puede conllevar su retirada del hogar familiar por parte del Instituto Colombiano de Bienestar Familiar. Debido a la imperante cultura patriarcal, la ausencia de los progenitores es más marcada a nivel paterno y esto se acentúa en las comunidades más empobrecidas del Estado Colombiano debido al asesinato de numerosos varones en los conflictos armados.

4. La falta de apoyo institucional, que se ve reflejada en la ausencia de oficinas de Bienestar Familiar, asesoría jurídica o puntos de salud pública en el barrio y la comuna. Este abandono acentúa la cronificación de las problemáticas familiares. Las familias se encuentran en una situación de aislamiento; los vínculos externos a su núcleo son escasos, inestables y en gran parte de los casos, contraproducentes.

5. La pobreza generalizada, que atraviesa a las familias transversalmente, e indirectamente incrementa la probabilidad de negligencia o maltrato intrafamiliar. En comunidades sin recursos de crianza, marcadas por la violencia, se da un mayor número de variables que contribuyen al estrés parental, el aislamiento social, la monoparentalidad y los embarazos adolescentes (Woodward \& Fergusson, 2002).

Tanto la ausencia de apoyo institucional como la pobreza generalizada ya mencionadas, son una evidencia más de la negligencia del Estado en su ejercicio de poder.

\section{Fouccuult: sobre territorio, estado y, poder}

Michael Foucault concibe el poder como objeto de acción, no de posesión. No hay grupos sociales 0 instituciones que "tengan el poder", sino que se ejerce de manera transversal y relacional en la sociedad. En las sociedades modernas la función del Estado es coordinar, proteger y gestionar las relaciones de poder entre otros (redes familiares, empresariales etc.). Así, el poder no se localiza puntualmente, sino que permanece difuso en los agentes sociales (Foucault, 1994).

En el contexto colombiano el manejo y control del poder por parte del Estado constituye un problema estructural. Este desprotege y manipula en su beneficio las relaciones de poder a nivel micro mediante la estigmatización de la pobreza, que se asocia a la violencia, convirtiéndola en una amenaza para el resto de la ciudadanía. Como consecuencia surgen las desigualdades en el acceso y distribución de recursos públicos entre la población de estratos más bajos que terminan por ser interiorizadas, aceptadas y convertidas en un estado psicológico de indefensión aprendida.

\section{Resiliencia: la esperanza ante la adversidad}

Emerge así el eje transversal de la presente investigación: las habilidades de afrontamiento ante las adversidades y las estrategias de resiliencia comunitarias. La resiliencia no es una cualidad interna e intrínseca que dependa de la actitud individual ante las dificultades, sino que, dentro de 
las subjetividades particulares, se ve afectada en gran parte por las circunstancias socio-históricas (económicas, políticas y familiares). Por otra parte, no surge de manera innata ante circunstancias desfavorables, sino que debe haber una posibilidad de cambio. Sin adversidad no puede haber resiliencia; sin esperanza tampoco (Frankl, 2004).

Frente a la extendida creencia de que una infancia infeliz aboca el desarrollo hacia comportamientos y rasgos de personalidad patológicos, la investigación realizada con menores resilientes ha probado que no existe evidencia científica de que una infancia desestructurada implique una adultez llena de fracasos (Cyrulnik, 2002). La capacidad de mantener un funcionamiento físico y psicológico adaptativo en situaciones críticas no es absoluta ni imperecedera, sino que resulta de un proceso dinámico y evolutivo modulable en función de las circunstancias, el contexto y la etapa vital, y cuya expresión depende de las diferentes culturas o entornos (Carretero Bermejo, 2010).

Al hablar de resiliencia y posibilidades de desarrollo, se deben evitar definiciones reduccionistas que consideran el desarrollo únicamente como incremento de ingresos personales, industrialización, avance tecnológico, modernización social, o crecimiento del PIB (Sen, 2000). Partimos del concepto de Desarrollo Humano, planteado por Amartya Sen, como un proceso de expansión de las libertades humanas reales, considerando también factores de bienestar social y político.

Este proceso requiere de la eliminación de importantes fuentes de ausencia de libertad como: pobreza y tiranía, escasas oportunidades económicas, privaciones sociales sistemáticas e intolerancia 0 sobreactuación de estados represivos (Sen, 2000). Todas ellas están presentes en el barrio de María Cano Carambolas en donde las libertades reales de sus habitantes están altamente restringidas. Estas libertades y la democracia, en la que los individuos son sujetos activos en la consecución del desarrollo, son esenciales para su avance (Charry Morales, 2010). Ambas son evaluadores de progreso; cuantas más libertades, más posibilidades de desarrollo; ya que el libre albedrío es un potente motor para este.

\section{Desaurrollo}

El objetivo de este artículo de reflexión es crear un diálogo en torno a las acciones de resistencia y estrategias de resiliencia de los vecinos de María Cano Carambolas, tras una estancia de observación participante e intervención comunitaria tanto en el centro educativo (Institución Educativa Reino de Bélgica) como en el barrio. Dicha observación fue documentada mediante diarios de campo y enmarcada en una investigación de corte cualitativo. La intervención realizada se entiende en la línea conceptual de Amalio Blanco, como un compromiso con los problemas sociales y reales; mediando e intercediendo en una determinada realidad y promoviendo la participación en el cambio (Blanco Abarca \& Rodríguez Marín, 2007).

Para la investigación se aunaron técnicas interactivas de investigación social cualitativa, que abarcaban la observación participante y los diarios de campo. La observación directa comprendió un período de cuatro meses, desde agosto hasta el fin del semestre en noviembre, con grupos de 
estudiantes de entre 8 y 16 años. El paradigma de observación participante involucra la interacción social entre el investigador y los informantes clave (Taylor \& Bogdan, 1984) durante la cual se recogen datos de modo sistemático y no intrusivo. Implica la selección de un escenario social, el barrio María Cano Carambolas; el acceso al mismo, en este caso a través del programa de intervención Cultura Amigó de la Universidad Católica Luis Amigó; y la interacción con los responsables de las organizaciones que favorecen dicho acceso, el director de la Institución Educativa y el responsable del Cultura Amigó. Dentro del barrio los informantes clave para esta investigación fueron la psicóloga de I.E.R.B, el alumnado y miembros de la JAC del barrio. Se utilizó una observación participante de tipo no estructurada, la más adecuada cuando el problema que se desea investigar no está muy acotado; es decir, no hay una definición previa sobre qué fenómenos 0 conductas vamos a observar y cómo se van a valorar, categorizar, medir o cuantificar. Este tipo de abordaje permite realizar una observación exploratoria y buscar la variabilidad de fenómenos y conductas que puedan ser de interés para el objeto de investigación.

A lo largo del contacto de los investigadoras con los informantes clave y de la estancia en el barrio, se desarrolló un registro a través de notas de campo donde se incluyeron descripciones de las situaciones cotidianas, la estructura del espacio físico y las interacciones sociales (entre las familias del barrio, la escuela y la JAC). A partir de la presencia en reuniones y conversaciones que se daban tanto en el núcleo escolar (con el equipo docente y directivo, la psicóloga y tutorías familiares) como en el barrio (con la JAC y miembros del proyecto Cultura Amigó) se pudo extraer información sobre la situación real del barrio y su evolución histórica.

Dentro de la I.E.R.B y junto con la psicóloga, se realizaron una serie de talleres psicoeducativos, como parte del programa de educación complementaria del centro. Las sesiones se llevaban a cabo durante el horario escolar, con una extensión de hora y media y abarcaban salones de 30 a 40 estudiantes. En los inicios de la intervención se realizaron talleres de temáticas diversas (corporalidad, autoconcepto, sexualidad, equidad de género, convivencia, prevención de bullying) a través de los cuales se estableció un vínculo entre investigadoras y los menores, lo que en observación participante se conoce como rapport.

Una vez adquirido cierto nivel de confianza con el alumnado, se procedió a centrar el foco de los talleres en la exploración y profundización en sus experiencias de resiliencia con los estudiantes de edades comprendidas entre 12 y 15 años. Se incluyeron de manera oral relatos que encarnaban conductas resilientes para contextualizar el ejercicio. El objetivo era que posteriormente pudiesen exteriorizar situaciones propias o cercanas que para ellos implicaran un abordaje desde la resiliencia. Para ello, se les pidió que redactaran en tercera persona un pequeño relato sobre las cuestiones exploradas en las dinámicas anteriores a través del diálogo. Además de estos relatos, se les solicitó que definiesen, con sus propias palabras y sin previa explicación, qué significaba para ellos el concepto de resiliencia. De forma complementaria, se evaluaron las proyecciones a futuro, mediante una dinámica acerca de metas a medio y largo plazo. 
El análisis de los relatos y la información recogida en los talleres fue un proceso dinámico y creativo con el fin de obtener una comprensión más profunda. Primero tuvo lugar una fase de descubrimiento en la cual se identificó qué temas comunes emergen de los relatos. Para ello, se subrayó y recogió el contenido más significativo en relación con el objetivo de la investigación, con el propósito de darle un nombre a modo de categorías. Una vez destacada la información más pegada al texto, lo más próximo a los participantes, se procedió a agrupar las categorías por ejes temáticos más globales: Expectativas de futuro, Contexto escolar, Contexto familiar y Red de apoyo externo. A partir de estos escritos, se llevó a cabo una reflexión centrada en las habilidades de afrontamiento de la juventud del barrio. En segundo lugar, se puso en contexto la información recogida con las observaciones participantes de manera paralela en el barrio y en la escuela, para obtener un enfoque más global.

\section{Resiliencia apreciada en el allumnado}

Las condiciones promotoras de conductas resilientes, es decir, el acceso a recursos y oportunidades de desarrollo, parten de una base sólida de formación y educación que a estos jóvenes les ha sido negada. De los registros que surgieron durante la observación en la I.E.R.B se destaca que existen frecuentes paros del profesorado debido a los recortes de recursos por parte del Estado; de modo que las clases se cancelan y se reorganizan con escaso margen de tiempo. Como consecuencia se produce un descontrol en la asistencia y en los horarios; los menores se suelen preguntar "iHoy hay clase?", "iAyer asististe?".

Muchos no llevan regularmente las tareas hechas a clase o se olvidan de sus materiales escolares, y un gran número de ellos suspende el curso. Nos encontramos con abundantes faltas de ortografía, pensamiento muy concreto a edades avanzadas y atención dispersa. No obstante, incluso con estas condiciones, observamos que todo el personal del centro tiene un gran sentido de pertenencia al mismo. A pesar del escaso capital humano de la institución desde el personal de limpieza hasta el director, se coordinan e implican de manera ejemplar, anteponiendo incluso la educación de los alumnos y el buen funcionamiento del colegio a su horario laboral.

Es destacable poner en contexto que, en el momento de realizar los registros de los diarios de campo del alumnado, estos se encontraban en una época de elevado estrés y presión ya que el curso estaba a punto de finalizar y se aproximaban las evaluaciones. Durante las últimas clases, a diferencia de en la etapa inicial de observación, apreciamos mayor silencio en el aula, respeto del turno de palabra, alta participación y rendimiento individual y apoyo entre compañeros.

A continuación, Se transcribe una selección de los relatos más representativos, para el posterior análisis en función de las líneas temáticas encontradas:

Relato A: “Resiliencia: Había una vez una familia y tenían un hijo. Un día perdió a su mamá y a su papá entonces él se quedó solo y él no sabía qué hacer porque él pensaba que se iba a morir de hambre. Entonces pasó un mes y a él ya se le había acabado la comida, entonces él ya se le 
estaban acabando los sueños de ser empresario y tenía que salir a pedir monedas o comida en la calle. Y llegó una señora y le daba comida todos los días y la señora un día le dijo que si se iba a vivir con ella y él respondió que sí. Entonces ella le dijo que cuál era su sueño y él le respondió: ser empresario. Y él se sintió muy feliz porque la señora le dijo que ella lo va a meter a estudiar para cuando sea grande ser empresario. Pasaron 10 años y él ya era todo un empresario y estaba todo feliz".

Relato B: “Mi vida no es como pensaba: Un niño llamado Esteban pensaba que la vida era color de rosas porque tenía a su mamá y a su papá. Y aunque no los valoraba tanto, él los amaba mucho. Un día sus dos padres murieron. A él le dolió demasiado, pero se dio moral a él mismo y empezó a estudiar. Fue un gran empresario e hizo resiliencia en él mismo y reaccionó de una forma muy bonita y sus padres quedaron muy orgullosos de su gran hijo".

Relato C: “Problemas en la escuela: Un día como otro cualquiera, estaban los estudiantes de una academia. En esta siempre hay muchos problemas y de todos los tipos por todas partes; en el jardín, en los salones, en la cafetería... ipor todas partes! Hay un niño llamado Johnny que es nuevo en la escuela y ha sido internado 2 veces. Él también era problemático y siempre peleaba y robaba. Pasaban semanas y la situación no cambiaba en la academia. Johnny poco a poco fue entendiendo lo que sucedía en la academia y fue intentando remediar las cosas tratando bien a los demás y aconsejándolos y poco a poco todo mejoraba. A los días ya todo era más pacífico y amoroso, gracias a Johnny las cosas están bien y la academia se había mejorado".

Relato D: “Cómo enfrentar los problemas II: Un día un niño llamado Diego tenía el problema de que le hacían bullying; lo maltrataban y le pegaban. Entonces un día a Diego le dio mucha rabia con los que le pegaban y él le puso la queja al profesor, pero este no le paró bolas. Entonces fue a ponerle la queja a la mamá y esta sí se preocupó y fue a hablar al colegio para pedir explicaciones de por qué no le habían parado bolas. Pero todavía continuaron haciéndole bullying y un día Diego fue a confrontarlos y le puso fin al problema porque lo enfrentó con el diálogo y la escucha".

Relato E: “Niño problema: Érase una vez un niño que siempre peleaba y nadie lo quería y su mamá le pegaba todos los días y no tenía amigos. Él hacía bromas e iba perdiendo el año. Su mamá y todos sus compañeros. Su papá no vivía con ellos. Y un día el niño decidió hacer muchos amigos, pero lo rechazaron. Fue a la psicóloga y ella lo ayudó a él y a su mamá. Él fue bueno en el colegio, ganó el año y tuvo muchos amigos que lo respetaban y querían. Su mamá no le volvió a pegar y vivieron felices para siempre".

Relato F: “La resiliencia: Una familia muy feliz, vivía bien, todo estaba bien. Un día el papá del niño falleció en una moto, así que hicieron lo siguiente. La madre no podía más que llorar, los hijos estaban tristes y los hijos dejaron de estudiar por tal fraude que pasó. La mamá lo había superado pero los hijos no. Se quería suicidar el hijo menor. Fue tal la tragedia que su madre lo intenta calmar para que no se suicidara. Una noche estaban todos durmiendo, el hijo se levantó, fue al baño, cogió muchas pastillas, se las intenta comer, pero apareció el espíritu del papá. Le dijo: No te suicides. El espíritu desapareció, todos lo superaron, siguieron adelante y pudieron superarlo". 
Relato G: “Cómo enfrentar los problemas I: Había una vez una niña con muchos problemas con la familia y los amigos. Un día Ivana le dijo a su madre:

-Mamá yo quiero dejar todos esos problemas atrás y comenzar una vida nueva.

La mamá le dijo:

-Siempre que tengas problemas vete a la reja de detrás de la casa y clava un clavo.

Al día siguiente, la niña clavó 20 clavos y tenía 10 problemas. Pasaron 2 años y la niña había clavado 100 clavos. Y le dijo a la mamá:

-Ma, ya no tengo más problemas.

Y la mamá le dijo:

-Empieza a quitarlos y cuando tengas una alegría, quitas un clavo.

En 60 días ya lo había quitado y llevó a la mamá atrás y le dijo:

-Mira hija, la reja es como tu corazón; lleno de huecos y sin alegría.

La niña le dijo:

-Ma, ya aprendí la lección, te amo.

\section{Línea temática 1: expectativas de futuro}

Una de las metas comunes que se percibieron en el alumnado es el éxito académico. Mediante la ansiedad experimentada y manifestada acerca de sus estudios, apreciamos la preocupación por aprobar el curso y las posibles repercusiones en caso de no conseguirlo a nivel familiar y/o escolar. Incluso se pudo observar casos de estudiantes que acuden a la consulta de psicología con problemas de ansiedad por ir perdiendo el curso y no saber cómo comunicárselo a sus padres. Esto indica la importancia que le otorgan a la valoración del entorno familiar.

Gran parte del alumnado expresa su deseo a largo plazo de acceder a la educación universitaria y convertirse en "alguien en la vida". Mediante estas prácticas se van configurando formas y alternativas de futuro alejadas del barrio y diferentes a las de su entorno más próximo. Consecuentemente surgen y se retroalimentan nuevas mentalidades, aspiraciones y, en definitiva, nuevos sujetos. Por tanto, se percibe una resistencia a tener un futuro de pobreza, fracaso académico y profesional; es decir, a la exclusión social. Esta actitud, más o menos consciente dependiendo de la edad, se interpreta como una forma de agentividad y acción para conseguir las metas a las que aspiran y transformar el futuro que por contexto les viene asignado.

En los relatos de los menores se encontraron referencias de familiares y allegados que han alcanzado objetivos profesionales reconocidos por su prestigio social en el imaginario colectivo, como emprender en el mundo empresarial o ejercer en el ámbito médico. Concretamente, tanto en 
el relato $A$ como $B$, ambos atravesados por la ausencia parental, apreciamos ese sueño de partida de ser empresario y la consecución de dicha meta en el futuro. Los dos escritos dejan en evidencia la ambición y la creencia de ser capaces de conseguirlo. En el relato $A$ se expresa a través de los deseos del protagonista de "convertirse en empresario", mediados por el apoyo económico de una tercera persona. Mientras que en el relato B emerge del esfuerzo a través del estudio para llegar a la misma meta.

En esta línea, también podemos contemplar la causalidad que el alumnado otorga a la acción de estudiar con el éxito, tanto profesional como personal. En el caso del relato $B$, este alcanza sus metas laborales tras "estudiar duro" $y$, en el relato $C$, el protagonista hace un esfuerzo estudiando y aprobando el curso con el objetivo de ganarse el respeto de sus iguales.

Además de los relatos, se tuvo la oportunidad de impartir una dinámica externa relacionada con las metas a medio-largo plazo del alumnado en una de las sesiones psicoeducativas. Se les hizo una pregunta muy concreta: "¿Cómo te ves dentro de 10 años?". Si bien algunos respondieron con acciones cotidianas como "divertirme, jugar, bailar, hacer deporte, tener mi cuarto organizado", 0 con deseos a corto plazo como "que llegue el fin de semana, ir de excursión"; la mayoría enfocó sus respuestas en objetivos a medio-largo plazo como los académicos: "estudiar, ganar el año, hacer las tareas, ir a la universidad"; profesionales: "ser doctora, modelo, futbolista, youtuber, diseñadora, bailarina"; y personales: "no buscar peleas, no ser grosera con mi mamá, hacerme novia del niño que me gusta, participar en una competición deportiva".

La predisposición al aprendizaje del alumnado, en un entorno adverso en el que la calidad precaria de la educación básica que reciben y las dificultades añadidas de la vida en el barrio suponen una traba en el acceso a la universidad pública, son un ejemplo de motivación.

\section{Línea temática 2: contexto escolar}

Más allá de los frecuentes paros, del absentismo escolar y de las problemáticas de accesibilidad y desplazamiento a la escuela, tanto el director como la psicóloga de la institución educativa nos reportan que dentro del propio colegio se producen situaciones de acoso, cyberbullying, cutting, 0 consumo de sustancias psicoactivas, llegando en casos extremos a tentativas de suicidio como se puede observar en extractos del relato $C$ donde apunta que "en la escuela siempre hay muchos problemas", el protagonista es "un chico problemático" que "ha sido internado dos veces" y que "siempre peleaba y robaba". 0 en el relato $D$ en el que el menor era víctima de bullying, "lo maltrataban y le pegaban". Pero también muestra un episodio de negligencia por parte del equipo docente ya que ese mismo chico "le puso una queja al profesor, pero éste no le paró bolas".

Durante la estancia de los investigadoras en el centro educativo, se observó que la psicóloga escolar es la responsable de atender los casos de urgencia, activar rutas de acción individualizadas y realizar un seguimiento exhaustivo, con consultas psicológicas semanales. Sorprendió la coordinación y comunicación entre los principales actores del centro educativo con respecto a las 
problemáticas que surgen en el día a día y el elevado número de horas que dedican mensualmente a la implementación de talleres psicoeducativos grupales. Cada grupo de clase participaba en uno de ellos por lo menos durante hora y media cada semana.

Además, con el objetivo de promover una buena convivencia y solventar los conflictos que pudiesen surgir entre el alumnado del centro, se había creado un servicio de mediación liderado por alumnos con un perfil conciliador y pacífico. Dicho grupo estaba dirigido y coordinado por la psicóloga, pero eran los propios menores quienes se encargaban de detectar posibles casos de bullying y/o conflictos convivenciales con el fin de diseñar una ruta de intervención.

La perspectiva global y holística de esta intervención psicoeducativa del centro escolar incluye al entorno familiar, aunque, en numerosos casos la principal dificultad radica en la baja o nula asistencia de padres y madres a las sesiones.

\section{Línea temática 3: contexto familiar}

Por un lado, llamó la atención el hecho de que en casi todos los relatos se hiciese mención a la pérdida de uno 0 dos progenitores. En los diversos encuentros con las familias del centro educativo y del barrio, se pudo registrar que, en la mayoría de casos, es el padre quien representa la figura ausente del núcleo familiar. Los motivos más comunes que explican esta casuística son: el abandono de sus responsabilidades parentales y la muerte. En los relatos $A, B$ y $F$ del alumnado se observa cómo describen hechos en esta dirección: "un día perdió a su mamá y su papá y se quedó solo", "un día sus dos padres murieron y a él le dolió demasiado" y "un día el papá del niño falleció en una moto". Es importante tener presente que los relatos son una herramienta proyectiva para trasladar experiencias personales o cercanas, ya que el nivel de realismo no se ajusta al desarrollo imaginativo de tan tempranas edades.

Además, se registra un elevado número de alumnas de esta comunidad que toman el rol de madres cuidadoras de sus hermanos y primos. Las labores de crianza y cuidado, así como las tareas domésticas, recaen principalmente sobre las mujeres, en especial en familias monoparentales, 0 con un progenitor ausente. El perfil predominante es el de mujeres jóvenes, entre 16 y 25 años, con un promedio de dos hijos.

Por otro lado, se observan ejemplos de casuísticas de violencia prolongada en el núcleo familiar, característica de las familias multiproblemáticas. Se pudo apreciar, tanto en las tutorías con las madres como con la psicóloga del centro educativo, que los núcleos familiares del barrio estaban marcados por la presencia de violencia de género y/o intrafamiliar, alcoholismo y/o consumo de sustancias psicoactivas, y autolesiones por parte de sus miembros. Los datos oficiales confirman la presencia de problemáticas que abarcan violencia intrafamiliar seguida de violencia sexual y desprotección de menores (Alcaldía de Medellín, 2014). Un gran número de relatos del alumnado hace mención a sucesos de esta índole, en el relato $E$ donde se describe que "su mamá le pegaba todos los días" o en el $\mathrm{G}$ hace explícita que la familia atravesaba situaciones difíciles. 
Además de los relatos, en diversos talleres los menores contaron que la forma de castigo más habitual que tenían tanto sus padres como sus madres era el uso de "correazos, cachetazos y gritos". Destacamos también en este apartado, un episodio en el que una de las alumnas, no acudió porque su padre la había sacado de la casa familiar junto a sus hermanas en mitad de la noche, tras una situación sostenida de conflicto con su pareja y madre de las niñas. Se trataba de una mujer de veinte años que llevaba soportando el maltrato físico y psicológico por parte de su ex pareja desde que tenía doce. Las hijas mayores empezaban a rebelarse contra ella, replicando los insultos de su pareja hacia ella.

La situación de precariedad vivida por las familias provoca que aspectos del desarrollo y la educación de los menores queden desatendidos. Las madres cuidadoras, cuya educación fue a su vez precaria, no disponen de los recursos y el tiempo suficientes para dar apoyo escolar a sus hijos. Así, como observamos día a día en la institución la mayoría no cuenta con el material escolar, el uniforme reglamentario y no siguen las medidas de higiene diarias.

\section{Línea temática 4: red de apoyo externo}

La presencia de un entorno de soporte es considerada por diversos modelos teóricos (Vanistendael, 1994; Grotberg, 1996) como factor protector y promotor del desarrollo de conductas resilientes. En varios relatos encontramos que la mediación de personas externas en las situaciones adversas descritas resulta clave para su abordaje y superación.

Así ocurre en el relato A en el que sin el apoyo económico (alimento, estudios, vivienda) de una persona externa, el protagonista no habría podido salir adelante. En el relato $E$ se describe una falta de esta red de apoyo "nadie lo quería", "decidió hacer muchos amigos, pero lo rechazaron" y cómo finalmente el punto de inflexión se produce a través de la ayuda de una psicóloga, presumiblemente la del centro educativo ya que es la única profesional de esta índole accesible en el barrio. En el relato $\mathrm{F}$ el punto de inflexión se produce cuando "apareció el espíritu del papá" que había fallecido en un accidente. Es esta aparición quien protege a la persona protagonista de las ideas suicidas que había comenzado a desarrollar durante el duelo por su padre. Este relato es evidencia del papel clave que desempeña la espiritualidad y creencias religiosas entre la comunidad.

Esta reflexión se sustenta también en una dinámica paralela, parte de uno de los talleres psicoeducativos, en la que se solicitó al alumnado "Enumerar 3 figuras de referencia" que los cuidan y protegen. Las respuestas fueron bastante uniformes, además de miembros de la familia, se hizo gran referencia a “Dios”, “Jehová" o “Jesús”. En el relato G también se parte de problemáticas en la red de amistades y de nuevo aparece el componente espiritual cuando la vía de superación de las dificultades es a través de seguir un ritual "siempre que tengas problemas vete a la reja de detrás de la casa y clava un clavo" y "cuando tengas una alegría, quitas un clavo". 


\section{Estrategias de affontamiento observadas en la comunidad}

Los diarios de campo derivados de la observación participante en María Cano Carambolas resultaron fundamentales para comprender el funcionamiento de la comunidad, más allá del contexto escolar, en la evaluación de la resiliencia en al ámbito vecinal.

Dentro de los factores que promueven la esperanza a nivel comunitario, se encuentra de nuevo la fe religiosa como soporte y estrategia de apoyo. A pesar de ser Colombia un estado cuya Constitución establece a partir de 1991 la separación entre Iglesia y Estado, la fe cristiana impuesta por los colonos españoles en 1499, sigue hoy impregnada en la rutina de sus habitantes. Observamos la presencia de numerosas iglesias en la ciudad, crucifijos en las clases, paneles con simbología religiosa en los autobuses, abalorios personales con figuras de vírgenes o santos (colgantes, pulseras) y figuras protegidas por altares en las calles (cuidadas con velas y flores).

Recogemos también en nuestros registros la presencia cotidiana en el vocabulario de la comunidad de expresiones que apelan a la voluntad de Dios en sus deseos o ruego; así como la práctica de la oración como recurso para abordar situaciones complejas: "rezo a Dios para que me dé paciencia en esos momentos", "le pido a la Virgen que mis hijos estén bien y se curen" o "cuando me estreso, me meto en el cuarto y rezo para que me de fuerzas y me calme" son algunos de los ejemplos. Así, percibimos que la fe religiosa y la espiritualidad parecen ser factores de protección que influyen en el desarrollo de comportamientos resilientes como ya se refleja en los relatos del alumnado y se defiende en teorías de autores clásicos (Cyrulnik, 2018). El hecho de creer en algo a lo que atribuirle una explicación de los fenómenos que escapan de nuestro entendimiento y control, facilita la comprensión de situaciones desfavorables permitiendo así gestionar las adversidades.

Por otro lado, observamos que gran parte de las problemáticas que afectan a los habitantes del barrio se ven acentuadas por las condiciones de inestabilidad extrema y pobreza en las que se encuentran. Tal y como expresan los miembros de la JAC y la documentación sobre los orígenes del barrio, la mayoría de las familias se han visto forzadas a dejar sus vidas, hogares y parte de sus miembros en sus regiones de origen, afectadas por el conflicto armado, y a resistir en este nuevo territorio (Aristizábal Botero et al., 2018). La firma de los Acuerdos de Paz de La Habana a finales del 2016 entre el Gobierno colombiano y los líderes de las FARC refleja un compromiso de reparación del daño a las víctimas que incluye la devolución de las tierras expropiadas en el ámbito rural por los guerrilleros. Por tanto, la posibilidad futura de que las familias regresen a sus lugares de origen refuerza la percepción del asentamiento en el barrio como un período temporal.

Debido a este carácter provisional y a la forzosa localización periférica, existen dificultades estructurales como la carencia de accesibilidad y de infraestructuras que faciliten las condiciones de movilidad. La propia configuración del barrio imposibilita la construcción de carreteras de doble vía y la señalización vial. Como consecuencia, las busetas quedan atascadas en múltiples ocasiones y los peatones se ven obligados a compartir calzada con los vehículos motorizados. Sin embargo, 
tanto el personal técnico del transporte público como los vecinos, se coordinan para regular el tráfico, la accesibilidad a las zonas más elevadas de la comuna y el suministro de alimentos y bienes de primera necesidad.

No hay espacio de parqueo para vehículos privados, que se encuentran fuera del poder adquisitivo y las prioridades de la mayoría de los habitantes, los cuales se desplazan en motocicletas y transporte público. Respecto a este, se pudo comprobar en los desplazamientos y confirmar con informantes clave, que no existen reducciones de tarifa en función del barrio o del estrato, como ocurre con otros servicios públicos de la ciudad como museos o teatros. Así se observa cómo los habitantes de la comuna recurren a recursos precarios, que ponen en riesgo su seguridad. Es común encontrar a tres o cuatro personas en una sola moto, a bebés en la parte delantera de la misma; circulación a altas velocidades; ausencia de respeto hacia los peatones; ausencia de uso de casco, etc. Si bien la Alcaldía de Medellín tiene campañas educativas por la ciudad tratando temas como la seguridad vial, a través de cartelería, encontramos que estas no llegan a los barrios de estrato más bajo.

En cuanto a los sistemas públicos ambientales y condiciones de salubridad también manifiestan necesidades insatisfechas (Alcaldía de Medellín, 2014). Se logró comprobar cómo la recogida de basura no funcionaba con regularidad, y ante la falta de colectores, se acumulan montones de desperdicios y residuos en los lindes de la calzada, arroyos y laderas, proliferando la llegada de roedores e insectos a la zona residencial. Teniendo en cuenta las graves deficiencias estructurales de la comuna, sus propios habitantes se ven obligados a gestionar los residuos priorizando la funcionalidad de su eliminación sin considerar factores ambientales o el cuidado del territorio.

Debido a la exclusión de las campañas municipales, en este caso de conciencia ambiental, empiezan a existir iniciativas comunitarias por el mantenimiento de los espacios comunes. Diversas paredes con inscripciones o murales de "Mi barrio es limpio" o carteles que prohíben tirar la basura en las fincas, muestran que, aunque no les lleguen los mensajes de la Alcaldía, ellos son capaces de construir los suyos propios para fomentar el cuidado del espacio compartido.

\section{Conclusiones}

La resiliencia comunitaria depende de factores como la autoestima colectiva, el sentido de comunidad o las redes de apoyo (Suárez Ojeda, 2008). Se concluye que en el barrio María Cano Carambolas existe esta unión comunitaria entre sus habitantes, pero no así al lugar, al territorio. La situación vital de estos es tan inestable que la mayoría no toman el barrio como un lugar donde establecerse de forma permanente. Se trata de un barrio de construcción bastante reciente donde cada año llegan nuevos habitantes que mantienen la esperanza de volver a sus tierras de origen. Aun así, se 
establecen abundantes redes de apoyo en los microsistemas comunales: familias y vecinos, que colaboran mutuamente en las tareas laborales y domésticas (cuidado de menores y dependientes) para facilitarse entre sí la supervivencia cotidiana y colectiva (Acevedo, 2002).

Como víctimas del desplazamiento forzado, los habitantes de María Cano Carambolas se apropiaron del territorio, trabajando desde sus posibilidades para la mejora de su calidad de vida y la satisfacción de sus necesidades básicas que el Estado, ausente, no cubre. Desde el tejido de redes sociales de apoyo, han logrado "hacer barrio" construyendo espacios de socialización comunitarios como la iglesia, la escuela, las plazas o el salón comunal, como manifiestan los responsables de la JAC y el director de la I.E.R.B. Son ellos los principales artífices en la construcción y dignificación de su territorio, actualmente en peligro por los macroproyectos urbanísticos de la Administración municipal, ya que se encuentran en el rango de acción del cinturón verde metropolitano (Universidad de Antioquia, 2016). Esto es otro ejemplo de la violencia estructural por parte del Estado, en este caso de la Gobernación de Antioquia y la Alcaldía de Medellín, que priorizan y anteponen la recaudación y acumulación de riqueza para una minoría frente al bienestar de la ciudadanía más desfavorecida y vulnerable.

Esta comunidad y en especial, sus mujeres, se autoorganizan para paliar las consecuencias de los factores que afectan a las familias multiproblemáticas. A través de las charlas informales con mujeres en las reuniones en la escuela, haciendo mercado en el barrio etc. se evidencia y destaca los fuertes lazos que existen entre ellas, quienes forman una comunidad de cuidadoras atendiendo a hijos de vecinas, amigas y familiares, e incluso amamantándolos en los casos de mujeres que padecen hipogalactia, es decir, que no producen leche materna suficiente.

En cuanto al sistema educativo, autores como Belth (1971) y Fernández (2013) entienden que este debe conocer el contexto social de su propia nación (en este caso, barrio) en el que se encuentra inmerso ya que las reformas educativas siempre están acompañadas de cambios políticos y económicos. En el sistema educativo colombiano no se aprecia una conexión con el contexto social ni un planteamiento democrático de educación para todos. Solo las clases media y alta pueden acceder a oportunidades educativas y laborales del mismo rango. En el caso concreto de Medellín, los informes reflejan que no se ha logrado reducir el nivel de desigualdad en años promedio de escolaridad por ingresos (Medellín Cómo Vamos, 2020). Este factor, añadido a la necesidad de las familias de que sus hijos colaboren en sus negocios en el barrio, provoca que una pequeña minoría de jóvenes de María Cano Carambolas acceda a la educación superior universitaria y consecuentemente al desarrollo de competencias que en última instancia mejorarán su calidad de vida.

Por otra parte, existe una ausencia de alternativas de desarrollo fuera del horario escolar, como bibliotecas públicas y espacios comunitarios de aprendizaje, lo cual supone un caldo de cultivo para que los jóvenes se involucren en actividades de riesgo para la integridad propia y ajena (Alcaldía de Medellín, 2014). Sin embargo, observamos cómo desarrollan sus propias estrategias para invertir el tiempo libre en acciones más productivas a través del deporte (organizando torneos de fútbol, partidas amistosas) o la música (formando grupos de baile y danza). Vivir en un entorno alejado del núcleo urbano, rodeado de naturaleza y animales, puede suponer un beneficio para ellos y favorecer 
el desarrollo de una mayor independencia, autosuficiencia y calidad del tejido de redes sociales de apoyo. Hay una gran vida comunitaria en las calles, comercios y viviendas, así como libertad de movimiento y libertad de juego en los menores.

Paralelamente al análisis de las dinámicas comunitarias, se ha examinado el papel del Estado Colombiano y sus políticas asistencialistas. Esta entidad pública no solo no ofrece medidas de protección y vías de desarrollo y bienestar integral a comunidades especialmente vulnerables, sino que fomenta la desigualdad. El impacto social causado por el conflicto armado y el desplazamiento forzoso no es reparado ya que, en el caso de la Alcaldía de Medellín, provoca y permite desahucios y derribos de viviendas por falta de licencia en estas comunas, agravando la situación de desarraigo de las familias y la inestabilidad en la comunidad.

Resulta irónico y paradójico hablar de licencias, cuando hablamos de gente que ha sido saqueada y obligada, en el mejor de los casos, a malvender sus tierras dejando atrás su lugar de pertenencia, de arraigo. Sin embargo, los habitantes no abandonan este nuevo territorio, sino que lo reconstruyen. Se destaca el caso de un hombre, de unos 60 años de edad, al que la Alcaldía le había tumbado su vivienda, habiéndose visto obligado a rescatar sus muebles, sus pocas pertenencias y sus placas de uralita, y almacenarlas hasta poder reconstruirla en otro sitio. Otro ejemplo son los vecinos del aledaño barrio Brisas del Edén, quienes asfaltan su propia calzada, ante la falta de medios y servicios. Así el Estado, ausente y negligente, dificulta deliberadamente el acceso a las posibilidades de desarrollo de la comunidad a la que olvida e invisibiliza.

A pesar de la ausencia del Estado, otras instituciones realizan proyectos en el barrio. En 2015 se celebró un festival audiovisual “Región, memoria y territorio del barrio María Cano Carambolas" impulsado por la Universidad de Antioquía y colectivos del sector, que consiguieron reunir a más de 300 asistentes. La Universidad Católica Luis Amigó también tiene varios proyectos de intervención social a través del grupo Cultura Amigó y Desarrollo del Bienestar Familiar. La comunidad agradece y acoge de manera muy positiva estas iniciativas que deberían estar impulsadas por otras fuentes públicas. La participación es alta, menores y adultos salen de sus casas motivados para llegar a los talleres que se organizan en la calle o en cualquier espacio facilitado por la JAC.

Se concluye que el Estado parece verse sobrepasado y no consigue abarcar la problemática social nacional en su magnitud. Sin embargo, se infiere que tal situación no se debe a la extensión 0 diversidad de esta, sino a un interés en la polarización de la riqueza y el incremento de la brecha que separa a las clases sociales. Con todo ello, recuperando la idea que vertebra el presente artículo de reflexión y tras analizar el material y las impresiones recogidas, se puede afirmar que se produce un desarrollo de estrategias resilientes y habilidades de afrontamiento en la comunidad del barrio María Cano Carambolas.

Colombia es un país con gran diversidad ideológica y cultural donde surgen necesidades disgregadas cuyo Gobierno no cubre. Cabría entonces la posibilidad de plantear un sistema político y social que dote de una mayor autonomía a la gobernabilidad de las regiones con el fin de fomentar la cercanía y el conocimiento por parte de la ciudadanía de los candidatos que finalmente adminis- 
trarán la vida pública de la comunidad. Consecuentemente, hipotetizamos que se produciría un mayor nivel de compromiso por parte de los gobernantes, así como el buen ejercicio de sus labores. Esto supondría a su vez una estrategia preventiva de una de las mayores problemáticas en el país: los altos niveles de corrupción (Transparencia Internacional, 2019).

El barrio María Cano Carambolas es un ejemplo de autogobierno ya que gran parte de la responsabilidad del funcionamiento del barrio recae en sus propios habitantes. Este planteamiento no sería posible sin una proyección esperanzadora del futuro, condición indispensable para el desarrollo personal y comunitario desde la resiliencia; citando de nuevo a Foucault, el poder va más allá de la política y la economía, pues está presente en todos los agentes sociales. Así, los puntos de resistencia se encuentran dentro de la red de poder: allí donde hay poder hay resistencia. Los habitantes del barrio generan resistencia y ejercen su poder ocupándose de aquellas tareas que son obligación del Estado.

\section{Conflicto de intereses}

Las autoras declaran la inexistencia de conflicto de interés con institución o asociación comercial de cualquier índole.

\section{Agradecimientos}

Este artículo de reflexión fue desarrollado gracias al apoyo técnico que brindó el profesor Edison Francisco Viveros Chavarría, en cuyo grupo de investigación se enmarca el artículo; así como con la colaboración de Hamilton de Jesús Fernández Vélez quien proporcionó el acceso al proyecto “Cultura Amigó" desarrollado en el barrio María Cano Carambolas. Agradecemos también a la Universidad Católica Luis Amigó y a la Universidad de Deusto por hacer posible el intercambio académico del que nace esta investigación.

\section{Referencias}

Acevedo, G. (2002). Logoterapia y resiliencia. Nous. Boletín de Logoterapia y Análisis Existencial, 1(6), 23-40. http://www.logoterapia.net/uploads/06_acevedo_2002_reiliencia.pdf 
Alcaldía de Medellín (2014). Plan de Desarrollo Local-Comuna 3, Manrique. https://bit.ly/3wMUqWZ

Alcaldía de Medellín \& Universidad de Antioquia. (2014). El goce efectivo de los DDHH desde las dimensiones de la seguridad humana de la población juvenil en 12 barrios de las comunas 1,3 y 8 de Medellín y sistemas de información locales. https://issuu.com/medellinjoven/docs/ informe-investigacion-final-octubre

Aristizábal Botero, C. A., Cárdenas Avendaño, 0. M., \& Rengifo González, C. J. (2018). Desplazamiento, trayectorias y poblamiento urbano. El caso de la Comuna 3 Manrique, Medellín, 1970-2010. Estudios Políticos, (53), 126-147. https://doi.org/10.17533/udea.espo.n53a06

Belth, M. (1971). La educación como disciplina científica. El Ateneo.

Blanco Abarca, A., \& Rodríguez Marín, J. (2007). Intervención psicosocial. Pearson Educación.

Carretero Bermejo, R. (2010). Resiliencia. Una visión positiva para la prevención e intervención desde los servicios sociales. Nómadas. Revista Crítica de Ciencias Sociales y Jurídicas, 27(3), 91-103. https://www.redalyc.org/pdf/181/18113757004.pdf

Censo División Política de Medellín. (2018). Registraduría para las Elecciones Presidenciales de 2018 y Acta E24 Municipal. https://www. registraduria.gov.co/

Charry Morales, A. (2010). El concepto de desarrollo se orienta hacia lo humano. Revista Virtual de la Facultad de Psicología, 5, 1-53. https://bit.ly/3wMUqWZ

Cyrulnik, B. (2002). Los patitos feos: la resiliencia. Una infancia infeliz no determina la vida. Gedisa.

Cyrulnik, B. (2018). Psicoterapia de Dios: la fe como resiliencia. Gedisa.

Fernández, M. (2013). Análisis del contexto educativo en el contexto social. En M. J. Carrasco, J. Coronel, S. Fernández, M. García, S. González, \& E. Moreno. (2013), Conocer y comprender las organizaciones educativas: una mirada a las cajas chinas (pp. 42-55). Ediciones Pirámide.

Foucault, M. (1994). El orden del discurso. Tusquets.

Frankl, V. (2004). El hombre en busca del sentido. Herder.

Gómez, E., \& Kotliarenco, M.A. (2010). Resiliencia familiar: un enfoque de investigación e intervención con familias multiproblemáticas. Revista de Psicología, 19(2), 103-131. 10.5354/0719-0581.2010.17112

Gómez, E., Muñoz, M. M., \& Haz, A. M. (2007). Familias multiproblemáticas y en riesgo social: características e intervención. Psykhe (Santiago), 16(2), 43-54. http://ojs.uc.cl/index.php/ psykhe/article/view/19899

Grotberg, E. H. (1996). The International Resilience Project: Resultados de la investigación y eficacia de las intervenciones. Actas de la 54ª Convención Anual del Consejo Internacional de Psicólogos. IC Press. 
Medellín Cómo Vamos. (2020). Informe de Calidad de Vida de Medellín 2016-2019. https://www. medellincomovamos.org/calidad-de-vida/ informe-de-calidad-de-vida

Phillips, D. A., \& Shonkoff, J. P. (Eds.) (2000). From neurons to neighborhoods: The science of early childhood development. National Academies Press. https://doi.org/10.17226/9824

Sen, A. (2000). El desarrollo como libertad. Gaceta Ecológica, (55), 14-20. https://www.redalyc. org/pdf/539/53905501.pdf

Suárez Ojeda, E. N. (2008). Una concepción latinoamericana: la resiliencia comunitaria. En A. Melillo \& E. N. Suárez Ojeda (Comps.), Resiliencia: Descubriendo las propias fortalezas (pp. 67-82). Paidós.

Taylor, S. J., \& Bogdan, R. (1984). La observación participante en el campo. En J. T. Stephen \& R. Bogdan (Eds.), Introducción a los métodos cualitativos de investigación. La búsqueda de significados (pp. 50-99). Paidós Ibérica.

Transparencia Internacional. (2019). Índice de percepción de la corrupción. https://transparenciacolombia.org.co/wp-content/ uploads/cpi2019-report-es-web-1.pdf

Universidad CES \& Secretaría de Salud de Medellín. (2012). Primerestudio poblacional de saludmental Medellín, 2011-2012. http://www.odc.gov.co/ Portals/1/publicaciones/pdf/consumo/estudios/ locales/C0031052011-primer-estudio-poblacional-de-salud-mental-medellin,-2011-2012. pdf

Universidad de Antioquia. (2016). Tejiendo los hilos de la memoria: historia local de Medellín desde los pobladores de la periferia. Comunas 3, 6 y 8, período 1970-2014. https://bibliotecadigital. udea.edu.co/bitstream/10495/11055/1/PerezAndrea_2015_TejiendoHilosMemoria.pdf

Vanistendael, S. (1994). La resiliencia: un concepto largo tiempo ignorado. BICE.

Woodward, L. J., \& Fergusson, D. M. (2002). Parent, child and contextual predictors of childhood physical punishment. Infant and Child Development: An International Journal of Research and Practice, 11(3), 213-235. https://doi.org/10.1002/icd.252 